\title{
ON A PIECE OF HYPERSURFACE IN A RIEMANNIAN MANIFOLD WITH MEAN CURVATURE BOUNDED AWAY FROM ZERO
}

\author{
BY \\ YOSHIE KATSURADA $\left({ }^{1}\right)$
}

Introduction. In 1965, S. S. Chern proved the following theorem:

THEOREM 0.1. Let $M$ be a compact piece of an oriented hypersurface of dimension $m$ with smooth boundary $\partial M$, which is immersed in an euclidean space $E$ of dimension $m+1$. Suppose the mean curvature $H_{1} \geqq c>0$. Let $a$ be a fixed unit vector which makes an angle $\leqq \pi / 2$ with the normals of $M$. Then

$$
m c V_{a} \leqq L_{a}
$$

where $V_{a}$ is the volume of the orthogonal projection of $M$ and $L_{a}$ that of $\partial M$ in the hyperplane perpendicular to a. If $M$ is defined by the equation

$$
z=z\left(x_{1}, \ldots, x_{m}\right), \quad x_{1}^{2}+\cdots+x_{m}^{2} \leqq R^{2},
$$

where $x_{1}, \ldots, x_{m}, z$ are rectangular coordinates in the space $E$ and the $z$-axis is chosen in the direction of $a$, then $c R \leqq 1[1, \mathrm{p} .82]\left({ }^{2}\right)$.

This theorem is a generalization of Heinz's theorem for the $C^{2}$-surface defined by an equation $z=z(x, y)$ in euclidean three space [2].

The purpose of this paper is to extend this theorem to a hypersurface in a Riemann manifold.

The author wishes to express to Professor Shiing Shen Chern her sincere thanks for his valuable advice and suggestions, and also to Professor Shoshichi Kobayashi for his kind help.

1. A certain generalization of Chern's theorem. We consider a Riemann manifold $R^{m+1}(m+1 \geqq 3)$ of class $C^{\nu}(\nu \geqq 3)$ which admits a one-parameter group $G$ of transformations generated by an infinitesimal transformation:

$$
\bar{x}^{i}=x^{i}+\xi^{i}(x) \delta \tau
$$

(where $x^{i}$ are local coordinates in $R^{m+1}$ and $\xi^{i}$ are the components of a contravariant vector $\xi$. If $\xi$ is a Killing vector, a homothetic Killing vector, a conformal Killing vector, etc. [3, p. 32], then the group $G$ is called isometric, homothetic, conformal, etc.

Received by the editors May 10, 1966.

(1) Partially supported by NSF Grant GP-3990.

(2) Numbers in brackets refer to the references at the end of the paper. 
In $R^{m+1}$, we consider a domain $D$. If the domain $D$ is simply covered by the paths of the transformations generated by $\xi$, and $\xi$ is everywhere of class $C^{v}$ and $\neq 0$ in $D$, then we call $D$ a regular domain with respect to the vector field $\xi$.

In a regular domain $D$, we choose a coordinate system such that the paths of the transformations generated by $\xi$ are new $x^{1}$-coordinate curves, that is, a coordinate system in which the vector $\xi$ has the components $\delta_{1}^{i}$ (where $\delta_{j}^{i}$ denotes the Kronecker delta). Then (1.1) becomes $\bar{x}^{i}=x^{i}+\delta_{1}^{i} \delta \tau$ and the domain $D$ admits the transformation given by

$$
\bar{x}^{i}=x^{i}+\delta_{1}^{i} \tau .
$$

Now we consider a compact piece $M$ of an oriented hypersurface of dimension $m$ with smooth boundary $\partial M$, which is immersed in a regular domain $D$ with respect to the vector field $\xi$. The immersion $x: M \rightarrow R^{m+1}$ is locally given by

$$
x^{i}=x^{i}\left(u^{\alpha}\right), \quad i=1, \ldots, m+1, \quad \alpha=1, \ldots, m .
$$

Here and henceforth, Latin indices run from 1 to $m+1$ and Greek indices from 1 to $m$. Let us consider a hypersurface which is orthogonal to the new $x^{1}$-coordinate curves and project $x(M)$ along the $x^{1}$-coordinate curve into the orthogonal hypersurface, and let $M^{\prime}$ be the image of $M$. If $x^{\prime}(p)$ is the image of $x(p), p \in M$ under this projection, we have

$$
x^{\prime i}=x^{i}\left(u^{\alpha}\right)-\delta_{1}^{i} \tau\left(u^{\alpha}\right)
$$

where $\tau$ is a function defined on $M$.

Let us consider a differential form of degree $m-1$ at a point $p$ of $M$, defined by

$$
\left(\left(n,\left(\rho^{\prime} / \rho\right)^{m-1}\left(\delta_{1} / \rho\right), d x, \ldots, d x\right)\right) \stackrel{\text { def. }}{=} g^{1 / 2}\left(n,\left(\rho^{\prime} / \rho\right)^{m-1}\left(\delta_{1} / \rho\right), d x, \ldots, d x\right),
$$

where the symbol $($ ) means a determinant of order $m+1$ whose columns are the components of respective vectors or vector-valued differential forms, $n$ is a unit normal vector at a point $p$ of $M, d x$ is a displacement along $M, g$ is the determinant of the metric tensor $g_{i j}$ of $R^{m+1}$, and the functions $\rho$ and $\rho^{\prime}$ are defined by

$$
\begin{gathered}
\rho(p)=\text { length of the vector } \delta_{1} \text { at } x(p)=\left(g_{11}(x(p))\right)^{1 / 2}, \\
\rho^{\prime}(p)=\text { length of the vector } \delta_{1} \text { at } x^{\prime}(p)=\left(g_{11}\left(x^{\prime}(p)\right)\right)^{1 / 2} .
\end{gathered}
$$

Then the exterior differential of the differential form (1.4) divided by $m$ ! becomes

$$
\begin{aligned}
& \frac{1}{m !} d\left(\left(n,\left(\rho^{\prime} / \rho\right)^{m-1} \delta_{1} / \rho, d x, \ldots, d x\right)\right) \\
& \quad=\frac{1}{m !}\left\{\left(\left(D n,\left(\rho^{\prime} / \rho\right)^{m-1} \delta_{1} / \rho, d x, \ldots, d x\right)\right)+\left(\left(n, D\left(\rho^{\prime m-1} / \rho_{m} \delta_{1}\right), d x, \ldots, d x\right)\right)\right\}
\end{aligned}
$$

where the symbol $D$ means the covariant differential. 
The first term of the right-hand member of the above expression becomes

$$
\frac{1}{m !}\left(\left(D n,\left(\rho^{m-1} / \rho^{m}\right) \delta_{1}, d x, \ldots, d x\right)\right)=(-1)^{m} H_{1}\left(\rho^{\prime m-1} / \rho^{m}\right) n_{i} \delta_{1}^{i} d V
$$

where $H_{1}$ is the first mean curvature of $M$ and $d V$ is the volume element of $M$. On the other hand, we easily obtain the following relation:

$$
n_{i} \delta_{1}^{i} d V=\frac{(-1)^{m}}{m !}\left(\left(\delta_{1}, d x, \ldots, d x\right)\right)
$$

and from (1.3) we have

$$
\begin{aligned}
\frac{(-1)^{m}}{m !}\left(\left(\delta_{1}, d x, \ldots, d x\right)\right) & =\frac{g^{1 / 2}}{g^{1 / 2}} \frac{(-1)^{m}}{m !} g^{1 / 2}\left(\delta_{1}, d x^{\prime}, \ldots, d x^{\prime}\right) \\
& =\frac{g^{1 / 2}}{g^{\prime 1 / 2}} n_{i}^{\prime} \delta_{1}^{i} d V_{\xi}^{\prime}
\end{aligned}
$$

where $n^{\prime}$ and $d V_{\xi}^{\prime}$ mean the unit normal vector and the volume element at the corresponding point $x^{\prime}(p)$ of $M^{\prime}$ respectively and $g^{\prime}=g\left(x^{\prime}\right)$. Consequently from (1.7) and (1.8), we have

$$
\begin{aligned}
\frac{\rho^{\prime m-1}}{\rho^{m}} n_{i} \delta_{1}^{i} d V & =\frac{g^{1 / 2}}{\left(g^{\prime}\right)^{1 / 2}}\left(\frac{\rho^{\prime}}{\rho}\right)^{m} \frac{\delta_{1}^{i}}{\rho^{\prime}} n_{i}^{\prime} d V_{\xi}^{\prime} \\
& =\frac{g^{1 / 2}}{\left(g^{\prime}\right)^{1 / 2}}\left(\frac{\rho^{\prime}}{\rho}\right)^{m} d V_{\xi}^{\prime},
\end{aligned}
$$

because $\delta_{1} / \rho^{\prime}$ is nothing but the unit normal vector of $M^{\prime}$.

Now we suppose that the transformation group $G$ is conformal. Then $R^{m+1}$ admits the infinitesimal transformation given by (1.1) with the additional condition

$$
\xi_{i: j}+\xi_{j: i}=2 \phi(x) g_{i j}
$$

where the symbol ";" means the covariant derivative. In the new coordinate system of the domain $D$, the additional condition becomes

$$
\frac{\partial g_{i j}}{\partial x^{1}}=2 \phi(x) g_{i j}
$$

Therefore the metric tensor $g_{i j}$ with respect to the new coordinate system has the form

$$
g_{i j}=\exp \left(2 \int \phi(x) d x^{1}\right) f_{i j}\left(x^{2}, \ldots, x^{m+1}\right)
$$

From this, we can easily deduce the following result:

$$
\frac{g^{1 / 2}}{\left(g^{\prime}\right)^{1 / 2}}\left(\frac{\rho^{\prime}}{\rho}\right)^{m}=\exp \left(\int_{x^{\prime 1}}^{x^{1}} \phi(x) d x^{1}\right)
$$


since $\rho=\left(g_{11}\right)^{1 / 2}$ and $\rho^{\prime}=\left(g_{11}^{\prime}\right)^{1 / 2}$. Therefore from (1.9) and (1.11), (1.6) becomes

$$
\frac{1}{m !}\left(\left(D n,\left(\rho^{\prime m-1} / \rho^{m}\right) \delta_{1}, d x, \ldots, d x\right)\right)=(-1)^{m} H_{1} \exp \left(\int_{x^{\prime 1}}^{x^{1}} \phi d x^{1}\right) d V_{\xi}^{\prime}
$$

Put

$$
v_{i}=\frac{\rho^{\prime m-1}}{\rho^{m}} \delta_{1}^{j} g_{i j}
$$

Then the second term of the right-hand member of (1.5) may be written as follows:

$$
\frac{1}{m !}\left(\left(n, D\left(\left(\rho^{m-1} / \rho^{m}\right) \delta_{1}\right), d x, \ldots, d x\right)\right)=\frac{(-1)^{m}}{2 m} L_{v} g_{i j} g^{* i j} d V
$$

where $L_{v} g_{i j}$ is the Lie derivative of the tensor $g_{i j}$ with respect to $v^{i}, g^{* i j}$ means $\left(\partial x^{i} / \partial u^{\alpha}\right)\left(\partial x^{j} / \partial u^{\beta}\right) g^{\alpha \beta}$, and $g_{\alpha \beta}$ is the metric tensor of $M$.

Accordingly from (1.12) and (1.14), we can rewrite (1.5) as follows:

$$
\begin{aligned}
& \frac{(-1)^{m}}{m !} d\left(\left(n,\left(\rho^{\prime} / \rho\right)^{m-1} \delta_{1} / \rho, d x, \ldots, d x\right)\right) \\
& \quad=\exp \left(\int_{x^{\prime 1}}^{x^{1}} \phi d x^{1}\right) H_{1} d V_{\xi}^{\prime}+\frac{1}{2 m} L_{v} g_{i j} g^{* i j} d V .
\end{aligned}
$$

Integrating both members of (1.15) over $M$ and applying Stokes' theorem we have

$$
\begin{aligned}
\frac{(-1)^{m}}{m !} \int_{\partial M}\left(\left(n,\left(\rho^{\prime} / \rho\right)^{m-1}\right.\right. & \left.\left.\delta_{1} / \rho, d x, \ldots, d x\right)\right) \\
= & \int_{M} \exp \left(\int_{x^{\prime 1}}^{x^{1}} \phi d x^{1}\right) H_{1} d V_{\xi}^{\prime}+\frac{1}{2 m} \int_{M} L_{v} g_{i j} g^{* i j} d V .
\end{aligned}
$$

On the other hand, from (1.3) and (1.11) we can see that

$$
\begin{aligned}
\frac{(-1)^{m}}{m !}\left(\left(n,\left(\rho^{\prime} / \rho\right)^{m-1}\right.\right. & \left.\left.\delta_{1} / \rho, d x, \ldots, d x\right)\right) \\
= & \frac{(-1)^{m}}{m !} \frac{g^{1 / 2}}{\left(g^{\prime}\right)^{1 / 2}}\left(\frac{\rho^{\prime}}{\rho}\right)^{m}\left(g^{\prime}\right)^{1 / 2}\left(n, \delta_{1} / \rho^{\prime}, d x^{\prime}, \ldots, d x^{\prime}\right) \\
= & \frac{(-1)^{m}}{m !} \exp \left(\int_{x^{\prime}}^{x^{1}} \phi d x^{1}\right)\left(g^{\prime}\right)^{1 / 2}\left(n, \delta_{1} / \rho^{\prime}, d x^{\prime}, \ldots, d x^{\prime}\right) .
\end{aligned}
$$

Now for each point $p \in M$, let us take the small piece $\tilde{M}_{p}$ of the hypersurface passing through the corresponding point $x^{\prime}(p)$ of $M^{\prime}$, given by the following expression

$$
\tilde{x}^{i}=x^{i}\left(u^{\alpha}\right)-\delta_{1}^{i} \tau_{p}, \quad \tau_{p}=\tau(p)=\text { const, }
$$

where $\tau_{p}$ means $x^{1}(p)-x^{\prime 1}(p)$. Then we have

$$
\frac{\partial \tilde{x}^{i}}{\partial u^{\alpha}}=\frac{\partial x^{i}}{\partial u^{\alpha}}
$$


and we can obtain the following relation:

$$
n_{\mathfrak{i}}=\frac{\left|\tilde{g}_{\alpha \beta}\right|^{1 / 2}}{\left|g_{\alpha \beta}\right|^{1 / 2}} \frac{g^{1 / 2}}{\left(g^{\prime}\right)^{1 / 2}} \tilde{n}_{\mathfrak{i}}
$$

where $\tilde{g}_{\alpha \beta}$ is the metric tensor of $\tilde{M}_{p}$ and $\tilde{n}$ is the unit normal vector of $\tilde{M}_{p}$ at $x^{\prime}(p)$, $\left|g_{\alpha \beta}\right|$ and $\left|\tilde{g}_{\alpha \beta}\right|$ mean the determinants of $g_{\alpha \beta}$ and $\tilde{g}_{\alpha \beta}$. Since

$$
g_{\alpha \beta}=g_{i j}(x) \frac{\partial x^{i}}{\partial u^{\alpha}} \frac{\partial x^{j}}{\partial u^{\beta}}, \quad \tilde{g}_{\alpha \beta}=g_{i j}\left(x^{\prime}\right) \frac{\partial \tilde{x}^{i}}{\partial u^{\alpha}} \frac{\partial \tilde{x}^{j}}{\partial u^{\beta}}
$$

making use of (1.10) we have

$$
\left|g_{\alpha \beta}\right|=\exp \left(2 m \int_{x^{\prime 1}}^{x^{1}} \phi d x^{1}\right)\left|\tilde{g}_{\alpha \beta}\right| .
$$

Therefore we have the following relation:

$$
n_{i}=\exp \left(\int_{x^{\prime 1}}^{x^{1}} \phi d x^{1}\right) \tilde{n}_{i}
$$

Since

$$
g_{i j}(x)=\exp \left(2 \int_{x^{\prime 1}}^{x^{1}} \phi d x^{1}\right) g_{i j}\left(x^{\prime}\right)
$$

it follows that

$$
g^{i j}(x)=\exp \left(-2 \int_{x^{\prime 1}}^{x^{1}} \phi d x^{1}\right) g^{i j}\left(x^{\prime}\right)
$$

Thus we obtain

$$
n^{i}=\exp \left(-\int_{x^{\prime 1}}^{x^{1}} \phi d x^{1}\right) \tilde{n}^{i}
$$

Substituting the above result in (1.17), we get

$$
\frac{(-1)^{m}}{m !}\left(\left(n,\left(\rho^{1} / \rho\right)^{m-1} \delta_{1} / \rho, d x, \ldots, d x\right)\right)=\frac{(-1)^{m}}{m !}\left(g^{\prime}\right)^{1 / 2}\left(\tilde{n}, \delta_{1} / \rho^{\prime}, d x^{\prime}, \ldots, d x^{\prime}\right)
$$

Let $e_{A}, A=1, \ldots, m-1$, be an oriented basis for the tangent plane of $\partial M^{\prime}$ at a point $x^{\prime}(p)$ on the image $\partial M^{\prime}$ of $\partial M$, and let $n^{\prime \prime}$ be the unit vector of the tangent plane of $M^{\prime}$ at the same point $x^{\prime}(p)$ which is orthogonal to $e_{A}, A=1, \ldots, m-1$, $n^{\prime}$ and such that

$$
\left(\left(n^{\prime \prime}, n^{\prime}, e_{1}, \ldots, e_{m-1}\right)\right)>0 .
$$

If $d x^{\prime}$ is a displacement along $\partial M^{\prime}$, then

$$
\frac{(-1)^{m}}{(m-1) !}\left(\left(n^{\prime \prime}, n^{\prime}, d x^{\prime}, \ldots, d x^{\prime}\right)\right)
$$

is the volume element of $\partial M^{\prime}$. The unit normal vector $\tilde{n}$ of $\tilde{M}_{p}$ at $x^{\prime}(p)$ can be expressed as follows:

$$
\tilde{n}=\alpha n^{\prime \prime}+\beta n^{\prime}+\sum_{A=1}^{m-1} \gamma^{A} e_{A},
$$


where $\alpha, \beta, \gamma^{A}$ satisfy

$$
\alpha^{2}+\beta^{2}+g_{i j}\left(x^{\prime}\right) e_{A}^{i} e_{B}^{j} \gamma^{A} \gamma^{B}=1 .
$$

Therefore we have $|\alpha| \leqq 1$. Since $\delta_{1} / \rho^{\prime}$ is the unit normal vector $n^{\prime}$, it follows that

$$
\frac{(-1)^{m}}{(m-1) !}\left(\left(\tilde{n}, \delta_{1} / \rho^{\prime}, d x^{\prime}, \ldots, d x^{\prime}\right)\right)=\frac{(-1)^{m}}{(m-1) !} \alpha\left(\left(n^{\prime \prime}, n^{\prime}, d x^{\prime}, \ldots, d x^{\prime}\right)\right) \text {. }
$$

By making use of a notation $d L_{\xi}^{\prime}$ for the volume element of $\partial M^{\prime}$, from (1.18) and (1.19), (1.17) becomes

$$
\frac{(-1)^{m}}{m !}\left(\left(n,\left(\rho^{\prime} / \rho\right)^{m-1} \delta_{1} / \rho, d x, \ldots, d x\right)\right)=\frac{1}{m} \alpha d L_{\xi}^{\prime}, \quad|\alpha| \leqq 1 .
$$

Substituting (1.20) in (1.16), we have

$$
\frac{1}{m} \int_{\partial M} \alpha d L_{\xi}^{\prime}=\int_{M} \exp \left(\int_{x^{\prime 1}}^{x^{1}} \phi d x^{1}\right) H_{1} d V_{\xi}^{\prime}+\frac{1}{2 m} \int_{M} L_{v} g_{i j} g^{* i j} d V .
$$

Consequently it follows that

$$
\frac{1}{m} \int_{\partial M} d L_{\xi}^{\prime} \geqq \int_{M} \exp \left(\int_{x^{\prime 1}}^{x^{1}} \phi d x^{1}\right) H_{1} d V_{\xi}^{\prime}+\frac{1}{2 m} \int_{M} L_{v} g_{i j} g^{* i j} d V .
$$

From the above inequality, we can obtain the following

THEOREM 1.1 (MAIN THEOREM). Let $M$ be a compact piece of an oriented hypersurface of dimension $m$ with smooth boundary $\partial M$, which is immersed in a regular domain of a Riemann manifold of dimension $m+1$ admitting a conformal Killing vector field $\xi$ (i.e., $\xi_{i ; j}+\xi_{j ; i}=2 \phi g_{i j}$ ). Suppose the mean curvature $H_{1} \geqq c>0$ ( $c=$ const). Let us suppose that the $\xi(x)$ makes an angle $\leqq \pi / 2$ with the normals of $M$, and let

$$
\begin{aligned}
\int_{x^{\prime}}^{x^{1}} \phi d x^{1} & \geqq 0 \quad \text { everywhere on } M, \\
\int_{M} L_{v} g_{i j} g^{* i j} d V \geqq 0 . &
\end{aligned}
$$

Then

$$
m c V_{\xi}^{\prime} \leqq L_{\xi}^{\prime}
$$

where $V_{\xi}^{\prime}$ and $L_{\xi}^{\prime}$ are the volumes of the images $M^{\prime}$ of $M$ and $\partial M^{\prime}$ of $\partial M$, by the projection along the paths of the vector field $\xi$ into a hypersurface perpendicular to the paths of $\xi$.

2. Some examples of the main theorem. In this section, we shall give some examples which satisfy the hypotheses (I) and (II) in the main theorem.

The hypothesis (I) means that either $\xi$ is a Killing vector or $\rho \geqq \rho^{\prime}$ everywhere on $M$. 
As an example in the case satisfying the hypothesis (II), we have the following

THEOREM 2.1. Let $R^{m+1}$ admit a conformal Killing vector field (i.e., $\xi_{i ; j}+\xi_{j ; i}$ $=2 \phi_{i j}$ ) and if the paths of the transformations generated by $\xi$ are geodesics (i.e., the new $x^{1}$-coordinate curves are geodesics), and $\phi \geqq 0$ everywhere on $M$, then it follows that

$$
L_{v} g_{i j} g^{* i j} \geqq 0
$$

everywhere on $M$.

Proof. Let us choose a coordinate system such that the family of hypersurfaces perpendicular to the new $x^{1}$-coordinate curves are new $x^{2} x^{3} \cdots x^{m+1}$-coordinate hypersurfaces. Then $g_{1 j}=0$ for $j=2, \ldots, m+1$.

Since the $x^{1}$-coordinate curve is a geodesic, substituting

$$
d x^{1} / d s=1 /\left(g_{11}\right)^{1 / 2}, d x^{2} / d s=\cdots=d x^{m+1} / d s=0
$$

in the differential equation of a geodesic:

$$
\frac{d^{2} x^{i}}{d s^{2}}+\Gamma_{j k}^{i} \frac{d x^{j}}{d s} \frac{d x^{k}}{d s}=0, \quad i=1,2, \ldots, m+1,
$$

we have

$$
-\frac{1}{2} \frac{1}{g_{11}} \frac{\partial g_{11}}{\partial x^{1}}+\Gamma_{11}^{1}=0, \quad \Gamma_{11}^{j}=0, \quad j=2, \ldots, m+1
$$

Substituting $\partial g_{i k} / \partial x^{1}=2 \phi g_{i k}$ in $\Gamma_{11}^{j}(j=2, \ldots, m+1)$, we obtain

$$
\Gamma_{11}^{j}=\frac{1}{2} g^{j k} \frac{\partial g_{11}}{\partial x^{k}}, \quad j=2, \ldots, m+1 .
$$

And also from $g^{j 1}=0, j=2, \ldots, m+1$, the conditions $\Gamma_{11}^{j}=0, j=2, \ldots, m+1$, become

$$
\sum_{k=2}^{m+1} g^{j k} \frac{\partial g_{11}}{\partial x^{k}}=0, \quad j=2, \ldots, m+1
$$

Since the determinant of $g^{j k}(j, k=2, \ldots, m+1)$ is not equal to zero, the conditions $\Gamma_{11}^{j}=0(j=2, \ldots, m+1)$ are rewritten as follows:

$$
\frac{\partial g_{11}}{\partial x^{k}}=0, \quad k=2, \ldots, m+1
$$

And the condition

$$
-\frac{1}{2} \frac{1}{g_{11}} \frac{\partial g_{11}}{\partial x^{1}}+\Gamma_{11}^{1}=0
$$

follows clearly from $g_{1 j}=0, j=2, \ldots, m+1$. Therefore in this case, a necessary and sufficient condition that the $x^{1}$-coordinate curve be a geodesic is that $\partial g_{11} / \partial x^{k}=0, k=2, \ldots, m+1$. 
To calculate $L_{v} g_{i,} g^{* i j}$, we go back to the definition (1.13):

$$
v_{i}=\frac{\rho^{m-1}}{\rho^{m}} \delta_{1}^{j} g_{i j}
$$

where $\rho=\left(g_{11}(x)\right)^{1 / 2}$ and $\rho^{\prime}=\left(g_{11}\left(x^{\prime}\right)\right)^{1 / 2} \cdot \rho^{\prime}$ is constant on the $x^{1}$-coordinate curve. Then we have

$$
\begin{aligned}
v_{i: j}= & \frac{1}{2} \frac{\partial g_{i j}}{\partial x^{1}} \frac{\left(g_{11}^{\prime}\right)^{(m-1) / 2}}{\left(g_{11}\right)^{m / 2}}-\frac{m}{2} \frac{\left(g_{11}^{\prime}\right)^{(m-1) / 2}}{\left(g_{11}\right)^{(m+2) / 2}} \frac{\partial g_{11}}{\partial x^{j}} \delta_{1}^{l} g_{i l} \\
& +\frac{m-1}{2} \frac{\left(g_{11}^{\prime}\right)^{(m-3) / 2}}{\left(g_{11}\right)^{m / 2}} \frac{\partial g_{11}^{\prime}}{\partial x^{j}} \delta_{1}^{l} g_{i l}, \\
L_{v} g_{i j}= & v_{i ; j}+v_{j: i} \\
= & 2 \phi g_{i j} \frac{\left(g_{11}^{\prime}\right)^{(m-1) / 2}}{\left(g_{11}\right)^{m / 2}}-\frac{m}{2} \frac{\left(g_{11}^{\prime}\right)^{(m-1) / 2}}{\left(g_{11}\right)^{(m+2) / 2}}\left(\left(\partial g_{11} / \partial x^{j}\right) g_{1 i}+\left(\partial g_{11} / \partial x^{i}\right) g_{1 j}\right) \\
& +\frac{m-1}{2} \frac{\left(g_{11}^{\prime}\right)^{(m-3) / 2}}{\left(g_{11}\right)^{m / 2}}\left(\left(\partial g_{11}^{\prime} / \partial x^{j}\right) g_{1 i}+\left(\partial g_{11}^{\prime} / \partial x^{i}\right) g_{1 j}\right)
\end{aligned}
$$

because $\partial g_{i j} / \partial x^{1}=2 \phi g_{i j}$. From the fact that the $x^{1}$-coordinate curves are geodesics and $\left(g_{11}^{\prime}\right)^{1 / 2}$ is constant on the $x^{1}$-coordinate curve, we have the following relations:

$$
\begin{array}{ll}
\frac{\partial g_{11}}{\partial x^{j}}=0, & j=2, \ldots, m+1, \\
\frac{\partial g_{11}^{\prime}}{\partial x^{1}}=0, \quad \frac{\partial g_{11}^{\prime}}{\partial x^{j}}=\frac{\partial g_{11}^{\prime}}{\partial x^{\prime j}}=0, & j=2, \ldots, m+1,
\end{array}
$$

since $x^{\prime j}=x^{j}, j=2, \ldots, m+1$. By making use of the above relations, we get

$$
\begin{aligned}
L_{v} g_{i j} g^{* i j} & =2 m \phi \frac{\left(g_{11}^{\prime}\right)^{(m-1) / 2}}{\left(g_{11}\right)^{m / 2}}-m \frac{\left(g_{11}^{\prime}\right)^{(m-1) / 2}}{\left(g_{11}\right)^{(m+2) / 2}} \frac{\partial g_{11}}{\partial x^{1}} g_{11} \frac{\partial x^{1}}{\partial u^{\alpha}} \frac{\partial x^{1}}{\partial u^{\beta}} g^{\alpha \beta} \\
& =2 m \phi \frac{\left(g_{11}^{\prime}\right)^{(m-1) / 2}}{\left(g_{11}\right)^{m / 2}}\left(1-g_{11} \frac{\partial x^{1}}{\partial u^{\alpha}} \frac{\partial x^{1}}{\partial u^{\beta}} g^{\alpha \beta}\right)
\end{aligned}
$$

and also we can see that

$$
g_{j k} \frac{\delta_{1}^{j}}{\left(g_{11}\right)^{1 / 2}} \frac{\partial x^{k}}{\partial u^{\alpha}}=\left(g_{11}\right)^{1 / 2} \frac{\partial x^{1}}{\partial u^{\alpha}},
$$

since the unit vector $\delta_{1}^{i} /\left(g_{11}\right)^{1 / 2}$ at a point on $M$ is expressed as follows:

$$
\frac{\delta_{1}^{i}}{\left(g_{11}\right)^{1 / 2}}=\lambda n^{i}+\mu^{\alpha} \frac{\partial x^{i}}{\partial u^{\alpha}}
$$

where

$$
\lambda^{2}+g_{i j} \frac{\partial x^{i}}{\partial u^{\alpha}} \frac{\partial x^{j}}{\partial u^{\beta}} \mu^{\alpha} \mu^{\beta}=\lambda^{2}+g_{\alpha \beta} \mu^{\alpha} \mu^{\beta}=1
$$


we have

$$
g_{i j} \frac{\delta_{1}^{i}}{\left(g_{11}\right)^{1 / 2}} \frac{\partial x^{j}}{\partial u^{\beta}}=g_{i j} \frac{\partial x^{i}}{\partial u^{\alpha}} \frac{\partial x^{j}}{\partial u^{\beta}} \mu^{\alpha}=g_{\alpha \beta} \mu^{\alpha} .
$$

Therefore it follows that

$$
g_{11} \frac{\partial x^{1}}{\partial u^{\alpha}} \frac{\partial x^{1}}{\partial u^{\beta}} g^{\alpha \beta}=g_{\alpha \gamma} \mu^{\gamma} g_{\beta \theta} \mu^{\theta} g^{\alpha \beta}=g_{\gamma \theta} \mu^{\gamma} \mu^{\theta} \leqq 1
$$

Consequently if $\phi \geqq 0$ everywhere on $M$, then it follows that

$$
L_{v} g_{i j} g^{* i j}=2 m \phi \frac{\left(g_{11}^{\prime}\right)^{(m-1) / 2}}{\left(g_{11}\right)^{m / 2}}\left(1-g_{11} \frac{\partial x^{1}}{\partial u^{\alpha}} \frac{\partial x^{1}}{\partial u^{\beta}} g^{\alpha \beta}\right) \geqq 0
$$

everywhere on $M$.

From Theorem 1.1 and Theorem 2.1, we can see

THEOREM 2.2. Let $M$ be a compact piece of an oriented hypersurface of dimension $m$ with smooth boundary $\partial M$, which is immersed in a regular domain of a Riemann manifold of dimension $m+1$ admitting a conformal Killing vector field $\xi$ with $\xi_{i ; j}+\xi_{j: i}=2 \phi g_{i j}, \phi \geqq 0$. Suppose the mean curvature $H_{1} \geqq c>0$ ( $c=$ const) and suppose that $\xi(x)$ makes an angle $\leqq \pi / 2$ with the normal at each point of $M$. If the paths of the transformations generated by $\xi$ are geodesics, then

$$
m c V_{\xi}^{\prime} \leqq L_{\xi}^{\prime}
$$

where $V_{\xi}^{\prime}$ and $L_{\xi}^{\prime}$ are the volumes of the image $M^{\prime}$ of $M$ and the image $\partial M^{\prime}$ of $\partial M$, by the projection along the paths into a hypersurface perpendicular to the paths, which never intersects $M$.

COROLlaRY 2.1. Let $M$ be a compact piece of an oriented hypersurface of dimension $m$ with smooth boundary $\partial M$, which is immersed in a regular domain of a Riemann manifold of dimension $m+1$ admitting a homothetic Killing vector field $\xi(x)$ with $\xi_{i: j}+\xi_{j: i}=2 c_{0} g_{i j}, c_{0}>0$, or a Killing vector field. Suppose the mean curvature $H_{1} \geqq c>0$ (c=const) and that $\xi(x)$ makes an angle $\leqq \pi / 2$ with the normal at each point of $M$. If the paths of the transformations generated by $\xi$ are geodesics, then

$$
m c V_{\xi}^{\prime} \leqq L_{\xi}^{\prime},
$$

where $V_{\xi}^{\prime}$ and $L_{\xi}^{\prime}$ are the volumes of the image $M^{\prime}$ of $M$ and the image $\partial M^{\prime}$ of $\partial M$, by the projection along the paths into a hypersurface perpendicular to the paths, which never intersects $M$.

Especially if our manifold $R^{m+1}$ is an euclidean space $E^{m+1}$ and if $\xi$ is the homothetic Killing vector field on $E^{m+1}$ with components $\xi^{i}=x^{i}, x^{i}$ being rectangular coordinates in the space $E^{m+1}$, then the paths of the transformations generated by 
$\xi$ are the lines through $O$ and a hypersurface perpendicular to these paths is a hypersphere of radius, say $R_{0}$, around $O$. The functions $\rho$ and $\rho^{\prime}$ are given by

$$
\rho=\left(\sum_{i=1}^{m+1}\left(x^{i}\right)^{2}\right)^{1 / 2} \text { and } \rho^{\prime}=R_{0} .
$$

Thus, as a special case of Corollary 2.1, we have the following

COROLLARY 2.2. Let $M$ be a compact piece of an oriented hypersurface of dimension $m$ with smooth boundary $\partial M$, which is immersed in an euclidean space of dimension $m+1$. Suppose that the position vector $x(u)$ makes an angle $\leqq \pi / 2$ with the normal of $x(M)$ at $x(u)$ for every $u \in M$ and that the mean curvature $H_{1}$ satisfies $H_{1} \geqq c>0$ (c= const). Then

$$
m c V_{\xi}^{\prime} \leqq L_{\xi}^{\prime}
$$

where $V_{\xi}^{\prime}$ and $L_{\xi}^{\prime}$ are the volumes of the image $M^{\prime}$ of $M$ and the image $\partial M^{\prime}$ of $\partial M$, by the projection from the origin $O$ into a hypersphere around $O$ not intersecting $M$.

From this result we have (under the same assumptions as Corollary 2.2)

COROllaRY 2.3. Let $\rho, \theta_{1}, \ldots, \theta_{m}$ be polar coordinates in $E^{m+1}$, i.e.,

$$
\begin{aligned}
& x^{1}=\rho \sin \theta_{m} \sin \theta_{m-1} \cdots \sin \theta_{2} \sin \theta_{1}, \\
& x^{2}=\rho \sin \theta_{m} \sin \theta_{m-1} \cdots \sin \theta_{2} \cos \theta_{1}, \\
& x^{3}=\rho \sin \theta_{m} \sin \theta_{m-1} \cdots \sin \theta_{3} \cos \theta_{2}, \\
& \vdots \\
& x^{m}=\rho \sin \theta_{m} \cos \theta_{m-1}, \\
& x^{m+1}=\rho \cos \theta_{m} .
\end{aligned}
$$

If $M$ is defined by the equation

then

$$
\rho=\rho\left(\theta_{1}, \ldots, \theta_{m}\right), \quad 0 \leqq \theta_{m} \leqq \phi_{0}(=\text { const }),
$$

$$
c C_{0}^{\prime} \leqq 1 \text {, }
$$

where $C_{0}^{\prime}$ is a constant which depends only on $R_{0}$ and $\phi_{0}\left(R_{0}\right.$ is the radius of the hypersphere into which $M$ is projected).

Proof. For simplicity, we shall prove in case $m=2$. Since $M$ is written as follows:

$$
\begin{aligned}
& x^{1}=\rho\left(\theta_{1}, \theta_{2}\right) \sin \theta_{2} \sin \theta_{1}, \\
& x^{2}=\rho\left(\theta_{1}, \theta_{2}\right) \sin \theta_{2} \cos \theta_{1}, \quad 0 \leqq \theta_{2} \leqq \phi_{0}, \\
& x^{3}=\rho\left(\theta_{1}, \theta_{2}\right) \cos \theta_{2},
\end{aligned}
$$

in this case, $M^{\prime}$ is defined by

$$
\begin{aligned}
& x^{1}=R_{0} \sin \theta_{2} \sin \theta_{1}, \\
& x^{2}=R_{0} \sin \theta_{2} \cos \theta_{1}, \quad 0 \leqq \theta_{2} \leqq \phi_{0}, \\
& x^{3}=R_{0} \cos \theta_{2},
\end{aligned}
$$


and $\partial M^{\prime}$ is the circle on the sphere $S_{0}$ with the center $O$ and radius $R_{0}$, given by

$$
\begin{aligned}
& x^{1}=R_{0} \sin \phi_{0} \sin \theta_{1}, \\
& x^{2}=R_{0} \sin \phi_{0} \cos \theta_{1}, \\
& x^{3}=R_{0} \cos \phi_{0} .
\end{aligned}
$$

Let $g^{*}$ be the determinant of the metric tensor $g_{\alpha \beta}^{*}$ on $S_{0}$ with respect to the coordinates $\theta_{1}, \theta_{2}$. Then $g^{*}=R_{0}^{4} \sin ^{2} \theta_{2}$. Therefore the area of $M^{\prime}$ is given by

$$
V_{\xi}^{\prime}=\int_{0}^{2 \pi} \int_{0}^{\phi_{0}} R_{0}^{2} \sin \theta_{2} d \theta_{2} d \theta_{1}=2 R_{0}^{2} \pi\left(1-\cos \phi_{0}\right)
$$

and the length of $\partial M^{\prime}$, which is a circle of radius $R_{0} \sin \phi_{0}$, is given by

$$
L_{\xi}^{\prime}=2 \pi R_{0} \sin \phi_{0} .
$$

From the results above, we have the following relation:

$$
2 V_{\xi}^{\prime}=2 R_{0} \frac{1-\cos \phi_{0}}{\sin \phi_{0}} L_{\xi}^{\prime}
$$

Putting

$$
C_{0}^{\prime}=2 R_{0} \frac{1-\cos \phi_{0}}{\sin \phi_{0}}
$$

and making use of Corollary 2.2, we obtain

$$
c C_{0}^{\prime} L_{\xi}^{\prime} \leqq L_{\xi}^{\prime}
$$

Thus $c C_{0}^{\prime} \leqq 1$.

Finally we consider a Killing vector field $\xi$ on $E^{m+1}$ which generates translations so that the paths of the transformations generated by $\xi$ are parallel lines in the direction of $\xi$ and a hypersurface perpendicular to these paths is a hyperplane perpendicular to $\xi$. In this case, Corollary 2.1 is nothing but Theorem 0.1 . Thus Chern's theorem is included as a special case in Corollary 2.1 .

\section{REFERENCES}

1. S. S. Chern, On the curvature of a piece of hypersurface in Euclidean space, Abh. Math. Sem. Univ. Hamburg 29 (1965), 77-91.

2. E. Heinz, Uber Flächen mit eineindeutiger Projektion auf eine Ebene, deren Krümmungen durch Ungleichungen eingeschränkt sind, Math. Ann. 129 (1955), 451-454.

3. K. Yano, The theory of Lie derivative and its applications, North-Holland, Amsterdam, 1957.

\footnotetext{
UNIVERSITY OF CALIFORNIA,

Berkeley, CaLifornia

HOKKAIDO UNIVERSITY,

SAPPORO, JAPAN
} 